\title{
Exploring Positive Noise in Estimation Theory
}

Kamiar Radnosrati, Gustaf Hendeby and Fredrik Gustafsson

The self-archived postprint version of this journal article is available at Linköping University Institutional Repository (DiVA):

http://urn.kb.se/resolve?urn=urn:nbn:se:liu:diva-167091

N.B.: When citing this work, cite the original publication.

Radnosrati, K., Hendeby, G., Gustafsson, F., (2020), Exploring Positive Noise in Estimation Theory, IEEE Transactions on Signal Processing, , 3590-3602. https://doi.org/10.1109/TSP.2020.2999204

Original publication available at:

https://doi.org/10.1109/TSP.2020.2999204

Copyright: Institute of Electrical and Electronics Engineers

http://www.ieee.org/index.html

(C)2020 IEEE. Personal use of this material is permitted. However, permission to reprint/republish this material for advertising or promotional purposes or for creating new collective works for resale or redistribution to servers or lists, or to reuse any copyrighted component of this work in other works must be obtained from the IEEE. 


\title{
Exploring Positive Noise in Estimation Theory
}

\author{
Kamiar Radnosrati, Gustaf Hendeby, Fredrik Gustafsson \\ Department of Electrical Engineering, Linköping University, Linköping, Sweden \\ Email: \{kamiar.radnosrati, gustaf.hendeby, fredrik.gustafsson\}@liu.se
}

\begin{abstract}
Estimation of the mean of a stochastic variable observed in noise with positive support is considered. It is well known from the literature that order statistics gives one order of magnitude lower estimation variance compared to the best linear unbiased estimator (BLUE). We provide a systematic survey of some common distributions with positive support, and provide derivations of minimum variance unbiased estimators (MVUE) based on order statistics, including BLUE for comparison. The estimators are derived with or without knowledge of the hyperparameters of the underlying noise distribution. Though the uniform, exponential and Rayleigh distributions, respectively, we consider are standard in literature, the problem of estimating the location parameter with additive noise from these distribution seems less studied, and we have not found any explicit expressions for BLUE and MVUE for these cases. In addition to additive noise with positive support, we also consider the mixture of uniform and normal noise distribution for which an order statisticsbased unbiased estimator is derived. Finally, an iterative global navigation satellite system (GNSS) localization algorithm with uncertain pseudorange measurements is proposed which relies on the derived estimators for receiver clock bias estimation. Simulation data for GNSS time estimation and experimental GNSS data for joint clock bias and position estimation are used to evaluate the performance of the proposed methods.
\end{abstract}

Index Terms-Order statistics, Estimation, Non-Gaussian noise, BLUE.

\section{INTRODUCTION}

We consider the problem of estimating a parameter $x$ observed in noise as $y_{k}=x+e_{k}$, for $k=1,2, \ldots, N$, also known as "estimation of location" [1], where the noise $e_{k}$ has positive support. We will refer to such distributions as positive noise. Examples of distributions we will study include the uniform, exponential, and Rayleigh distribution.

Problems involving positive noise can be motivated from applications where the arrival times of radio or sound waves are used. Such waves travel with the speed of the medium, and non-line of sight conditions give rise to delayed arrival times. This case occurs in a variety of applications such as target tracking using radar or lidar, and localization using radio waves as is done, for instance, in global navigation satellite systems (GNSS) [2, 3, 4, 5].

In the case of Gaussian noise, best linear unbiased estimator (BLUE) is given by the sample mean which gives the same weight to all observations. In these scenarios, the sample mean estimator coincides with the maximum likelihood estimator (MLE) and is optimal in Fisher's sense [6]. However, in many practical scenarios, the underlying noise is non-Gaussian resulting in a noticeable estimation error.

Radio network positioning, in which the unknown position of the target is estimated by measuring different properties of a wireless channel, is a motivating example in which the underlying noise is non-symmetric, skewed and nonGaussian [2,4]. This behavior is a result of one shared source of error, in addition to measurement noises, coming from propagation effects of the communication channel in harsh environments. While the conventional Kalman filter based estimators have the lowest mean squared error (MSE) among all possible solutions for Gaussian noise conditions [7], performance degradation in cluttered environments is inevitable. The achievable accuracy in such environments is included in e.g. [8]. In [9, 10,11], several variants of nonlinear filters such as particle filters are proposed to solve the nonlinear and non-Gaussian estimation problems occurring in target tracking and localization applications. Noting that particle filters might be over complicated for some applications, the authors in [12] consider a tradeoff between complexity and accuracy and propose a new Bayesian filtering solution well suited for nonlinear and non-Gaussian problems.

To deal with the estimation performance degradation for nonGaussian error conditions, conventional estimation techniques which are developed based on Gaussian assumptions need to be properly adjusted. As discussed in [13], "identify and discard" (see e.g., $[14,15,16]$ ), "mathematical programming" (see e.g., $[17,18,19])$, and "robust estimation" (see e.g., [20, 21]) are the three broad categories of estimation methods which are robust against non-Gaussian errors. Robustness of the estimator has long been a concern in both research [22] and engineering $[23,24,25]$. A recent survey on this topic can be found in [26].

The MLE, developed under Gaussian assumptions, can be modified to become robust in the presence of non-Gaussian noises. The authors in [27] first detect and then reject the outliers by learning the probability density function (PDF) of the measurements and developing a mixture model of outliers and clean data. A similar idea to the $k$-nearest-neighbor approach is used in [28] to classify outliers as the data points that are less similar to a number of neighboring measurements. Surveys of advances in clustering the data into outliers and clean data can be found in $[13,29,30]$. While these approaches might result in high estimation accuracy, they typically require large datasets [26].

Order statistics are known as powerful tools for providing simple, yet robust, estimators of location that can be used in different applications [31]. Although the theory of order statistics is well known in statistics literature, the application of order statistics to estimation problems, in particular localization problems, seems to have a large room for further investigations. Non-data aided channel estimation based on the first-order statistics for ultra wide-band communication is studied in [32]. Source localization using order statistics theory can also be 
found in $[33,34,35,36,37,38]$ for direct localization problems and in [39] for indirect localization problems. In all these studies, however, the sensor noise is assumed to have Gaussian distribution.

A bias compensated linear estimator as the sample mean has a variance that decays as $1 / N$, while it is well-known from the statistical literature, see for example [40,41], that picking the minimum observed value has a variance that decays as $1 / N^{2}$. The minimum value is the simplest example of order statistics. Certain care has to be taken for the cases where the parameters in the distributions are unknown, in which case bias compensation becomes difficult. This paper derives all combinations of known/unknown parameters for order statistics/BLUE for some selected and common distributions that allow for analytical solutions. The considered distributions are standard and their properties are well studied in literature $[40,41]$. However, we have not been able to find any explicit results for how to estimate a location parameter with additive noise from these distributions.

The rest of this paper is structured as follows. In Section II the estimation problem is formulated. The problem is then investigated for different noise distributions and estimators for each distribution are derived in Section III. The proposed estimators are evaluated in a simulation study for GNSS time estimation and the results are provided in Section IV. The estimators are further extended to fit the GNSS localization application and the results obtained from real GNSS data are given in Section $\mathrm{V}$ followed by the concluding remarks given in Section VI.

\section{Problem Formulation}

Consider the location estimation problem in which we have measurements $y_{k}, k=1, \ldots, N$ of the unknown parameter $x$. Assuming that the measurements are corrupted with additive noise $e_{k} \sim p_{e}(\theta)$, where $\theta$ denotes the parameter(s) of the noise distribution, the measurement model is given by

$$
y_{k}=x+e_{k}, \quad k=1, \ldots, N .
$$

The BLUE for the estimation problem (1) is given by

$$
\hat{x}_{\mathrm{BLUE}}^{p_{e}(\theta)}\left(y_{1: N}, \theta\right)=\frac{1}{N} \sum_{k=1}^{N} y_{k}-\delta(\theta) \text {, }
$$

where $y_{1: N}=\left\{y_{k}\right\}_{k=1}^{N}$ and $\delta(\theta)=\mathbb{E}\left(e_{k}\right)$ is the bias compensation term.

In addition to the BLUE, this work studies the problem of finding the minimum variance unbiased estimator (MVUE) for non-Gaussian noise distributions. It is worth mentioning that in the literature, the MVUE for some of the considered noise distributions are already derived, see [40, 41, 42]. However, these mainly focus on estimating the noise distribution parameter. This work surveys the MVUE, extended to the problem of estimating the mean $x$ observed in noise. It is worth noting that the MLE can also be defined for some of the considered cases. However, in general, a closed form expression for the estimator does not exist. For instance, in the case of uniformly distributed measurement noise with known hyperparameters, the MLE is ambiguous and given by any $x \in\left[\min _{k} y_{k}, \max _{k} y_{k}\right]$. For some other distributions, the MLE might be given by a constrained optimization problem. As we only present cases leading to analytical solutions and explicit MSE formulas, the MLE is not further discussed.

In order to find the MVUE, the first step is to find the PDF $f\left(y_{1: N} ; \theta\right)$, with $\theta$ denoting the parameters of the distribution. The Cramer-Rao lower bound (CRLB) theorem states that if the PDF fulfills the regularity condition

$$
\mathbb{E}\left[\frac{\partial \ln f\left(y_{k} ; \theta\right)}{\partial \theta}\right]=0 \quad \forall \theta,
$$

an unbiased estimator may be found that attains the bound for all $\theta$. Any unbiased estimator that satisfies CRLB is thus the MVUE. Note that (II) also implies that the support cannot depend on $\theta$. The distributions with positive support we consider do not satisfy the regularity condition and thus the CRLB approach cannot be used to determine if the estimators are MVUE. Instead, we rely on the Rao-Blackwell-LehmannScheffe (RBLS) theorem [43].

The RBLS theorem states that for any unbiased estimator $\tilde{x}$ and sufficient statistics $T\left(y_{1: N}\right), \hat{x}=\mathbb{E}\left(\tilde{x} \mid T\left(y_{1: N}\right)\right)$ is unbiased and $\operatorname{Var}(\hat{x}) \leq \operatorname{Var}(\tilde{x})$. Additionally, if $T\left(y_{1: N}\right)$ is complete, then $\hat{x}$ is an MVUE.

As shown in [40], if the dimension of the sufficient statistics is equal to the dimension of the parameter, then the MVUE is given by $\hat{x}=g\left(T\left(y_{1: N}\right)\right)$ for any function $g(\cdot)$ that satisfies

$$
\mathbb{E}(g(T))=x
$$

Hence, the problem of finding the MVUE becomes the problem of finding a complete sufficient statistic. The Neyman-Fisher theorem $[44,45]$ gives the sufficient statistic $T\left(y_{1: N}\right)$, if the PDF can be factorized as follows

$$
f\left(y_{1: N} ; \Psi\right)=g\left(T\left(y_{1: N}\right), \Psi\right) h\left(y_{1: N}\right),
$$

where $\Psi$ is the union of the noise hyperparameters $\theta$ and $x$. The estimators in this work are derived in the order statistics framework.

The marginal PDF $f_{(k, N)}(y)$ of the general $k$ th order statistics of a set of $N$ independent and identically random variables with common cumulative distribution function (CDF) $F(y)$ and $\operatorname{PDF} f(y)$ is given by

$$
f_{(k, N)}(y)=N f(y)\left(\begin{array}{c}
N-1 \\
k-1
\end{array}\right) F(y)^{k-1}(1-F(y))^{N-k} .
$$

See for instance [46] for a detailed explanation and derivations. In addition to BLUE and the MVUE, we also consider the minimum order statistic whose density $f_{(1, N)}(y)$ can be obtained by setting $k=1$ in (6)

$$
f_{(1, N)}(y)=N f(y)(1-F(y))^{N-1} .
$$

Let $\left(y_{(m)}\right)_{m=1}^{N}$ denote the ordered sequence obtained from sorting $y_{1: N}$ in an ascending order. The minimum order statistics estimator is given by

$$
\hat{x}_{\min }^{p_{e}(\theta)}\left(y_{1: N}\right)=y_{(1)} \triangleq \min _{k} y_{k} .
$$




\section{TABLE I: Notation.}

\begin{tabular}{l|l}
$\left\{y_{k}\right\}_{k=1}^{N}$ & $\begin{array}{l}\text { noisy measurements of the unknown parameter } x \\
\left(y_{(m)}\right)_{m=1}^{N}\end{array}$ \\
$\theta$ & $\begin{array}{l}\text { ordered measurement sequence } \\
\text { parameters of the noise distribution } \\
\text { bias compensation term }\end{array}$ \\
$\delta(\theta)$ & BLUE when $e_{k} \sim p_{e}$ for known $\theta$ \\
$\hat{x}_{\mathrm{BLUE}}^{p_{e}}\left(y_{1: N}, \theta\right)$ & $\begin{array}{l}\text { MVUE when } e_{k} \sim p_{e} \text { for known } \theta \\
\hat{x}_{\mathrm{MVUE}}^{p_{e}}\left(y_{1: N}, \theta\right) \\
\hat{x}_{\mathrm{MVUE}}^{p_{e}}\left(y_{1: N}\right)\end{array}$ \\
$\hat{x}^{p_{e}\left(y_{1: N}, \theta\right)}$ & $\begin{array}{l}\text { MVUE estimator when } e_{k} \sim p_{e} \text { for unknown } \theta \\
\hat{x}^{p_{e}}\left(y_{1: N}\right)\end{array}$
\end{tabular}

Noting that for any generic estimator $\hat{x}$ the MSE is given by

$$
\operatorname{MSE}(\hat{x})=\operatorname{Var}(\hat{x})+(\operatorname{bias}(\hat{x}))^{2} .
$$

The MSE for BLUE and MVUE or any other bias compensated estimator coincides with the estimator's variance. In the case of $\hat{x}_{\min }^{p_{e}(\theta)}\left(y_{1: N}\right)$, however, the existing bias enters the MSE. The theoretical MSE for the discussed estimators is also derived and provided in the following section.

Closed-form expressions of BLUE, MVUE and minimum order statistics estimators and their corresponding MSE for multiple noise distributions with positive support are provided in the sequel. In Section III, we distinguish between the estimators for which the underlying noise hyperparameter are known or unknown.

Given the hyperparameter $\theta$, the MVUE for each noise distribution $p_{e}$ is denoted by $\hat{x}_{\mathrm{MVUE}}^{p_{e}}\left(y_{1: N}, \theta\right)$. MVUE with unknown hyperparameter is denoted by $\hat{x}_{\mathrm{MVUE}}^{p_{e}}\left(y_{1: N}\right)$. If the MVUE cannot be found, an unbiased order statistics-based estimator is derived and denoted by $\hat{x}^{p_{e}}\left(y_{1: N}, \theta\right)$ and $\hat{x}^{p_{e}}\left(y_{1: N}\right)$ for the known and unknown hyperparameter cases, respectively. For example, $\hat{x}_{\text {MVUE }}^{\mathcal{U}}\left(y_{1: N}, \beta\right)$ denotes the MVUE when $e_{k} \sim$ $\mathcal{U}[0, \beta]$ and $\beta$ is known. $\hat{x}_{\text {MVUE }}^{\mathcal{U}}\left(y_{1: N}\right)$, on the other hand, corresponds to the MVUE of uniform noise with unknown hyperparameters of the distribution. Table I summarizes the notation used throughout this work.

\section{Estimators For Different NOISE Distributions}

In this section, estimators and their MSEs for a number of selected noise distributions are given. An extended list of distributions can be found in [47].

\section{A. Uniform distribution}

As the first scenario, consider the case in which the additive noise $e_{k}$ has a uniform distribution with a positive support. BLUE and MVUE of the uniform distribution parameters are derived in the literature (see e.g. [40]). Here, we derive estimators of $x$, given direct observations corrupted by uniformly distributed measurement noise with unknown hyperparamter. Let $p_{e}(\theta)=\mathcal{U}[0, \beta], \beta>0$ and $\theta=\beta$. The BLUE is given by

$$
\hat{x}_{\mathrm{BLUE}}^{\mathcal{U}}\left(y_{1: N}, \beta\right)=\frac{1}{N} \sum_{k=1}^{N} y_{k}-\frac{\beta}{2} \text {. }
$$

The MSE of BLUE, in this case, is equal to the variance of the estimator (10a) given by

$$
\operatorname{MSE}\left(\hat{x}_{\mathrm{BLUE}}^{\mathcal{U}}\left(y_{1: N}, \beta\right)\right)=\frac{1}{N^{2}} \sum_{k=1}^{N} \operatorname{Var}\left(y_{k}-\frac{\beta}{2}\right)=\frac{\beta^{2}}{12 N} .
$$

In order to find the MSE of the minimum order statistics estimator, $\hat{x}_{\min }^{\mathcal{U}}\left(y_{1: N}\right)$, we need to find the first two moments of the estimator. Let $\tilde{y}_{k}=\frac{1}{\beta} y_{k}$. Since $y_{k} \sim \mathcal{U}[x, x+\beta]$, then for any constant $\beta>0, \tilde{y}_{k} \sim \mathcal{U}\left[\frac{x}{\beta}, \frac{x}{\beta}+1\right]$. Hence, $f\left(\tilde{y}_{k}\right)=1$ and $F\left(\tilde{y}_{k}\right)=\frac{1}{\beta}\left(y_{k}-x\right)$ for $\tilde{y}_{k} \in\left[\frac{x}{\beta}, \frac{x}{\beta}+1\right]$ and zero otherwise. From (6) we get,

$$
\begin{gathered}
f_{(k, N)}^{\mathcal{U}[0, \beta]}(\tilde{y})=N\left(\begin{array}{c}
N-1 \\
k-1
\end{array}\right)\left(\frac{\tilde{y}-x}{\beta}\right)^{k-1}\left(\frac{\beta-(\tilde{y}-x)}{\beta}\right)^{N-k} \\
=\frac{(N) !}{(k-1) !(N-k) !}\left(\frac{\tilde{y}-x}{\beta}\right)^{k-1}\left(\frac{\beta-(\tilde{y}-x)}{\beta}\right)^{N-k} .
\end{gathered}
$$

Since $N \in \mathbb{N}^{+}, k \in \mathbb{N}^{+}$, and $k \in[1, N]$ we can the change the factorials to gamma functions,

$$
\begin{array}{r}
f_{(k, N)}^{\mathcal{U}[0, \beta]}(\tilde{y})=\frac{\Gamma(N+1)}{\Gamma(k) \Gamma(N-k+1)}\left(\frac{\tilde{y}-x}{\beta}\right)^{k-1} \\
\times\left(\frac{\beta-(\tilde{y}-x)}{\beta}\right)^{N-k} .
\end{array}
$$

The marginal distribution (11b) is a generalized beta distribution, also known as a four parameters beta distribution [48]. The support of this distribution is from 0 to $\beta>0$ and $f_{(k, N)}^{\mathcal{U}[0, \beta]}(\cdot)=\frac{1}{\beta} f_{(k, N)}^{\mathcal{U}[0,1]}(\cdot)$. The bias and variance of the minimum order statistics in the case of uniform noise with support on $[0, \beta]$ can then be derived and are given by

$$
\begin{aligned}
b\left(\hat{x}_{\min }^{\mathcal{U}}\left(y_{1: N}\right)\right) & =\frac{\beta}{N+1} \\
\operatorname{Var}\left(\hat{x}_{\min }^{\mathcal{U}}\left(y_{1: N}\right)\right) & =\frac{N \beta^{2}}{(N+1)^{2}(N+2)} .
\end{aligned}
$$

The MSE of $\hat{x}_{\min }^{\mathcal{U}}\left(y_{1: N}\right)$ is then given by

$$
\operatorname{MSE}\left(\hat{x}_{\min }^{\mathcal{U}}\left(y_{1: N}\right)\right)=\frac{2 \beta^{2}}{(N+1)(N+2)} .
$$

In order to find the MVUE, we note that the PDF can be written in a compact form using the step function $\sigma(\cdot)$ as

$$
f\left(y_{k} ; x, \beta\right)=\frac{1}{\beta}\left[\sigma\left(y_{k}-x\right)-\sigma\left(y_{k}-x-\beta\right)\right] .
$$

which gives

$$
\begin{gathered}
f\left(y_{1: N} ; x, \beta\right)=\frac{1}{\beta^{N}} \prod_{k=1}^{N}\left[\sigma\left(y_{k}-x\right)-\sigma\left(y_{k}-x-\beta\right)\right] \\
=\frac{1}{\beta^{N}}\left[\sigma\left(y_{(1)}-x\right)-\sigma\left(y_{(N)}-x-\beta\right)\right],
\end{gathered}
$$

where $y_{(N)} \triangleq \max _{k} y_{k}, \quad k=1, \ldots, N$. The expressions for the MVUE are derived for two different scenarios. We first assume that the hyperparameter $\beta$ of the noise distribution is known and then further discuss the unknown hyperparameter case. In the general case, let $\Psi=[x, \beta]^{\top}$ denote the 
unknown parameter vector, the Neyman-Fisher factorization gives $h\left(y_{1: N}\right)=1$ and

$$
T\left(y_{1: N}\right)=\left[\begin{array}{c}
y_{(1)} \\
y_{(N)}
\end{array}\right]=\left[\begin{array}{l}
T_{1}\left(y_{1: N}\right) \\
T_{2}\left(y_{1: N}\right)
\end{array}\right] .
$$

1) Known hyperparameter $\beta$ : When the maximum support of the uniform noise $\beta$ is known, the dimensionality of the sufficient statistic is larger than that of the unknown parameter $x$. As discussed in [40], the RBLS theorem can be extended to address this case if a function $g\left(T_{1}\left(y_{1: N}\right), T_{2}\left(y_{1: N}\right)\right)$ can be found that combines $T_{1}$ and $T_{2}$ into a single unbiased estimator of $x$.

Let $Z=T_{1}\left(y_{1: N}\right)+T_{2}\left(y_{1: N}\right)=u+v$. Since $T_{1}$ and $T_{2}$ are dependent,

$$
f_{Z}(z)=\int_{-\infty}^{\infty} f_{y_{(1)}, y_{(N)}}(u, z-u) d_{u}
$$

where $f_{y_{(1)}, y_{(N)}}(u, z-u)$ is the joint density of minimum and maximum order statistics. As shown in [49], for $-\infty<u<$ $v<\infty$, the joint density of two order statistics $y_{(i)}$ and $y_{(j)}$ is given by

$$
\begin{aligned}
f_{y_{(i)}, y_{(j)}}(u, v)= & \frac{N !}{(i-1) !(j-1-i) !(N-j) !} \\
& \times f_{Y}(u) f_{Y}(v)\left[F_{Y}(u)\right]^{i-1} \\
& \times\left[F_{Y}(v)-F_{Y}(u)\right]^{j-1-i}\left[1-F_{Y}(v)\right]^{N-j},
\end{aligned}
$$

that for the extreme orders, $i=1$ and $j=N$ can be simplified such that for $u<v$

$f_{y_{(1)}, y_{(N)}}(u, v)=N(N-1)\left[F_{Y}(v)-F_{Y}(u)\right]^{N-2} f_{Y}(u) f_{Y}(v)$.

and zero otherwise. Substituting (16c) into (16a), we get

$$
f_{Z}(z)=\frac{1}{2} N \beta^{-N}(2 x+2 \beta-z)^{N-1},
$$

for $2 x+\beta<z<2(x+\beta)$ and

$$
f_{Z}(z)=\frac{1}{2} N \beta^{-N}(z-2 x)^{N-1},
$$

for $2 x<z \leq 2 x+\beta$ and zero otherwise. It can be shown that

$$
\mathbb{E}\left(f_{Z}(z)\right)=2 x+\beta .
$$

Hence, noting that $\beta$ is known, the function $g\left(T_{1}\left(y_{1: N}\right), T_{2}\left(y_{1: N}\right)\right)$ that gives an unbiased estimator is

$$
\begin{aligned}
\hat{x}_{\mathrm{MVUE}}^{\mathcal{U}}\left(y_{1: N}, \beta\right) & =g\left(T_{1}\left(y_{1: N}\right), T_{2}\left(y_{1: N}\right)\right) \\
& =\frac{1}{2}\left(y_{(1)}+y_{(N)}\right)-\frac{\beta}{2} .
\end{aligned}
$$

The MSE of the MVUE is given by

$$
\operatorname{MSE}\left(\hat{x}_{\mathrm{MVUE}}^{\mathcal{U}}\left(y_{1: N}, \beta\right)\right)=\frac{\beta^{2}}{2 N(N+3)+4} .
$$

Compared to (10b), the order statistics based MVUE outperforms the BLUE by one order of magnitude.
2) Unknown hyperparameter $\beta$ : In the case of unknown hyperparameter, the MVUE for the parameter vector $\Psi=$ $[x, \beta]^{\top}$ can be derived from sufficient statistics (15),

$$
\hat{\Psi}=g\left(T\left(y_{1: N}\right)\right), \quad \text { s.t. } \quad \mathbb{E}\left(g\left(T\left(y_{1: N}\right)\right)\right)=\Psi .
$$

In this case, we have

$$
\mathbb{E}\left(T\left(y_{1: N}\right)\right)=\left[\begin{array}{l}
x+\frac{\beta}{N+1} \\
x+\frac{N \beta}{N+1}
\end{array}\right]
$$

To find the transformation that makes (19) unbiased, we define

$$
g\left(T\left(y_{1: N}\right)\right)=\left[\begin{array}{c}
\frac{1}{N-1}\left(N T_{1}\left(y_{1: N}\right)-T_{2}\left(y_{1: N}\right)\right) \\
\frac{N+1}{N-1}\left(T_{2}\left(y_{1: N}\right)-T_{1}\left(y_{1: N}\right)\right)
\end{array}\right]
$$

that gives

$$
\mathbb{E}\left(g\left(T\left(y_{1: N}\right)\right)\right)=\left[\begin{array}{l}
x \\
\beta
\end{array}\right] .
$$

Finally, the MVUE of $x$, when the hyperparameter $\beta$ is unknown, is given by

$$
\hat{x}_{\text {MVUE }}^{\mathcal{U}}\left(y_{1: N}\right)=\frac{N}{N-1} y_{(1)}-\frac{1}{N-1} y_{(N)} .
$$

and its MSE is

$$
\operatorname{MSE}\left(\hat{x}_{\mathrm{MVUE}}^{\mathcal{U}}\left(y_{1: N}\right)\right)=\frac{N \beta^{2}}{(N+2)\left(N^{2}-1\right)} .
$$

This is naturally slightly larger than (17b) for finite $N$.

\section{B. Distributions in the exponential family}

The exponential family of probability distributions, in their most general form, is defined by

$$
f(y ; \theta)=h(y) g(\theta) \exp \{A(\theta) \cdot T(y)\},
$$

where $\theta$ is the parameter of the distribution, and $h(y), g(\theta)$, $A(\theta)$, and $T(y)$ are all known functions. In this section, we only consider some examples of distributions of this family and show that the minimum order statistic estimator has the same form as the noise distribution but with modified parameters. For the selected distributions, if possible, MVUE for both cases of known and unknown hyperparameter are derived. Otherwise, unbiased estimators with less variance than BLUE are proposed.

1) Exponential distribution: The trend estimation in exponential noise is solved using a linear programming algorithm in [50] and a recursive maximum likelihood algorithm in [51]. The author in [52] proposes different estimators for functions of the noise distribution parameters. In the rest of this section, we derive BLUE and MVUE for the unknown parameter $x$ and the hyperparameters.The exponential PDF in terms of the scale parameter $\beta>0$ is given by

$$
f^{\operatorname{Exp}}\left(y_{k} ; x, \beta\right)=\left\{\begin{array}{cl}
\frac{1}{\beta} \exp \left(-\frac{\left(y_{k}-x\right)}{\beta}\right) & y_{k} \geq x, \\
0 & y_{k}<x .
\end{array}\right.
$$

and the $\mathrm{CDF}$, for $y_{k} \geq x$, is given by

$$
F^{\operatorname{Exp}}\left(y_{k} ; x, \beta\right)=1-\exp \left(-\frac{\left(y_{k}-x\right)}{\beta}\right) .
$$


From the properties of the exponential distribution, we directly get

$$
\hat{x}_{\mathrm{BLUE}}^{\operatorname{Exp}}\left(y_{1: N}, \beta\right)=\frac{1}{N} \sum_{k=1}^{N} y_{k}-\beta, \quad \operatorname{MSE}\left(\hat{x}_{\mathrm{BLUE}}^{\operatorname{Exp}}\right)=\frac{\beta^{2}}{N} .
$$

Substituting (23) into (6), the marginal density of the $k$ th order statistic is given by

$$
\begin{aligned}
f_{(k, N)}^{\operatorname{Exp}}(y ; x, \beta)=\frac{N}{\beta}\left(\begin{array}{c}
N-1 \\
k-1
\end{array}\right) & \left(1-\exp \left(-\frac{(y-x)}{\beta}\right)\right)^{k-1} \\
& \times \exp \left(-\frac{(N-k+1)(y-x)}{\beta}\right) .
\end{aligned}
$$

The first order statistic density is then given by letting $k=1$ in (25) which results in another exponential distribution,

$$
f_{(1, N)}^{\operatorname{Exp}}(y ; x, \beta)=f^{\operatorname{Exp}}(y ; x, \bar{\beta}),
$$

where $\bar{\beta}=\frac{\beta}{N}$. Hence, the MSE of the minimum order statistics estimator is given by

$$
\operatorname{MSE}\left(\hat{x}_{\min }^{\operatorname{Exp}}\left(y_{1: N}\right)\right)=\frac{2 \beta^{2}}{N^{2}} .
$$

In order to find the MVUE, we re-write the PDF as

$$
\begin{aligned}
f\left(y_{1: N} ; x, \beta\right) & =\frac{1}{\beta^{N}} \exp \left[-\frac{1}{\beta} \sum_{k=1}^{N} y_{k}\right] \exp \left[-\frac{N}{\beta} x\right] \\
& \times \sigma\left(y_{(1)}-x\right) .
\end{aligned}
$$

In the case of known hyperparameter $\beta$, the Neyman-Fisher factorization of PDF (28) gives

$$
\begin{aligned}
& T\left(y_{1: N}\right)=y_{(1)} \\
& h\left(y_{1: N}\right)=\frac{1}{\beta^{N}} \exp \left[-\frac{1}{\beta} \sum_{k=1}^{N} y_{k}\right] .
\end{aligned}
$$

The MVUE can then be obtained from a transformation of the minimum order statistic that makes it an unbiased estimator. It can be shown that the MVUE and its MSE are given by

$$
\begin{aligned}
\hat{x}_{M V U E}^{\operatorname{Exp}}\left(y_{1: N}, \beta\right) & =y_{(1)}-\frac{\beta}{N} \\
\operatorname{MSE}\left(\hat{x}_{M V U E}^{\operatorname{Exp}}\left(y_{1: N}, \beta\right)\right) & =\frac{\beta^{2}}{N^{2}} .
\end{aligned}
$$

If the hyperparameter $\beta$ is unknown, the factorization gives

$$
T\left(y_{1: N}\right)=\left[\begin{array}{c}
y_{(1)} \\
\sum_{k=1}^{N} y_{k}
\end{array}\right]=\left[\begin{array}{c}
T_{1}\left(y_{1: N}\right) \\
T_{2}\left(y_{1: N}\right)
\end{array}\right] .
$$

Noting that the sum of exponential random variables results in a Gamma distribution, we have $T_{2}\left(y_{1: N}\right) \sim \Gamma(N, \beta)$. Hence,

$$
\mathbb{E}\left(T\left(y_{1: N}\right)\right)=\left[\begin{array}{c}
x+\frac{\beta}{N} \\
N(x+\beta)
\end{array}\right] \text {. }
$$

Following the same line of reasoning as in Section III-A2, the unbiased estimator is given by the transformation

$$
g\left(T\left(y_{1: N}\right)\right)=\left[\begin{array}{c}
\frac{1}{N-1}\left(N T_{1}\left(y_{1: N}\right)-\frac{1}{N} T_{2}\left(y_{1: N}\right)\right) \\
\frac{1}{N-1}\left(T_{2}\left(y_{1: N}\right)-N T_{1}\left(y_{1: N}\right)\right)
\end{array}\right],
$$

that gives

$$
\mathbb{E}\left(g\left(T\left(y_{1: N}\right)\right)\right)=\left[\begin{array}{l}
x \\
\beta
\end{array}\right] .
$$

Finally, the MVUE when the hyperparameter $\beta$ is unknown is given by

$$
\begin{aligned}
\hat{x}_{\mathrm{MVUE}}^{\operatorname{Exp}}\left(y_{1: N}\right) & =\frac{N}{N-1} y_{(1)}-\frac{1}{N(N-1)} \sum_{k=1}^{N} y_{k} \\
& =\frac{N}{N-1} y_{(1)}-\frac{1}{N-1} \bar{y},
\end{aligned}
$$

where $\bar{y}$ is the sample mean. Assuming that $N$ is large, $\min _{k} y_{k}$ and $\bar{y}$ are independent and the MSE of the estimator, asymptotically, is given by

$$
\operatorname{MSE}\left(\hat{x}_{\mathrm{MVUE}}^{\operatorname{Exp}}\left(y_{1: N}\right)\right)=\frac{\beta^{2}(N+1)}{N(N-1)^{2}} .
$$

2) Rayleigh distribution: One generalization of the exponential distribution is obtained by parameterizing in terms of both a scale parameter $\beta$ and a shape parameter $\alpha$. The Rayleigh distribution is a special case obtained by setting $\alpha=2$. The location estimation problem in Rayleigh noise is rather an un-explored area. In this section, we derive BLUE and order statistics based unbiased estimators. The Rayleigh PDF is defined as

$$
f^{\text {Rayleigh }}\left(y_{k} ; x, \beta\right)=\left\{\begin{array}{cl}
\frac{y_{k}-x}{\beta^{2}} \exp \left(-\frac{\left(y_{k}-x\right)^{2}}{2 \beta^{2}}\right) & y_{k}>x \\
0 & y_{k} \leq x
\end{array}\right.
$$

and the $\mathrm{CDF}$, for $y_{k}>x$, is given by

$$
F^{\text {Rayleigh }}\left(y_{k} ; x, \beta\right)=1-\exp \left(-\frac{\left(y_{k}-x\right)^{2}}{2 \beta^{2}}\right) .
$$

For the BLUE, we have

$$
\begin{aligned}
\hat{x}_{\mathrm{BLUE}}^{\text {Rayleigh }}\left(y_{1: N}, \beta\right) & =\frac{1}{N} \sum_{k=1}^{N} y_{k}-\sqrt{\frac{\pi}{2}} \beta, \\
\operatorname{MSE}\left(\hat{x}_{\mathrm{BLUE}}^{\text {Rayleigh }}\left(y_{1: N}, \beta\right)\right) & =\frac{(4-\pi) \beta^{2}}{2 N} .
\end{aligned}
$$

The marginal density of the $k$ th order statistic, for $y>x$, is given by

$$
\begin{aligned}
f_{(k, N)}^{\text {Rayleigh }}(y ; x, \beta) & =\frac{N y}{\beta^{2}}\left(\begin{array}{c}
N-1 \\
k-1
\end{array}\right)\left(1-\exp \left(-\frac{(y-x)^{2}}{2 \beta^{2}}\right)\right)^{k-1} \\
& \times \exp \left(-\frac{(N-k+1)(y-x)^{2}}{2 \beta^{2}}\right)
\end{aligned}
$$

Hence, the minimum order statistics density is also Rayleigh distributed

$$
f_{(1, N)}^{\text {Rayleigh }}(y ; x, \beta)=f^{\text {Rayleigh }}(y ; x, \bar{\beta}),
$$

where $\bar{\beta}=\frac{\beta}{\sqrt{N}}$. The MSE of the minimum order statistics is given by

$$
\operatorname{MSE}\left(\hat{x}_{\min }^{\text {Rayleigh }}\left(y_{1: N}\right)\right)=\frac{2 \beta^{2}}{N}
$$


The joint PDF of $N$ independent observations $y_{1: N}$ is given by

$$
\begin{aligned}
f\left(y_{1: N} ; x, \beta\right) & =\frac{\prod_{k=1}^{N}\left(y_{k}-x\right)}{\beta^{2 N}} \exp \left[\sum_{k=1}^{N}-\frac{\left(y_{k}-x\right)^{2}}{2 \beta^{2}}\right] \\
& \times \sigma\left(y_{(1)}-x\right) .
\end{aligned}
$$

Noting that

$$
\sum_{k=1}^{N}\left(y_{k}-x\right)^{2}=\sum_{k+1}^{N} y_{k}^{2}-2 x \sum_{k=1}^{N} y_{k}+N x^{2},
$$

the PDF becomes

$$
\begin{aligned}
f\left(y_{1: N} ; x, \beta\right) & =\beta^{-2 N} \prod_{k=1}^{N}\left(y_{k}-x\right) \exp \left[\frac{-1}{2 \beta^{2}} \sum_{k=1}^{N} y_{k}^{2}\right] \\
& \times \exp \left[-\frac{N x^{2}}{2 \beta^{2}}\right] \exp \left[\frac{x}{\beta^{2}} \sum_{k=1}^{N} y_{k}\right] \sigma\left(y_{(1)}-x\right) .
\end{aligned}
$$

Since (39c) cannot be factorized in the form of $f\left(y_{1: N} ; x, \beta\right)=$ $g\left(T\left(y_{1: N}\right), x\right) h\left(y_{1: N}\right)$, the RBLS theorem cannot be used. Hence, even if an MVUE exists for this problem, we may not be able to find it. Thus, in the case of Rayleigh-distributed measurement noise, we propose unbiased estimators based on order statistics.

If the hyperparameter of the distribution is known, the unbiased order statistic estimator $\hat{x}^{\text {Rayleigh }}\left(y_{1: N}, \beta\right)$ is then given by

$$
\begin{aligned}
\hat{x}^{\text {Rayleigh }}\left(y_{1: N}, \beta\right) & =y_{(1)}-\frac{\sqrt{\pi} \beta}{\sqrt{2 N}}, \\
\operatorname{MSE}\left(\hat{x}^{\text {Rayleigh }}\left(y_{1: N}, \beta\right)\right) & =\frac{(4-\pi) \beta^{2}}{2 N} .
\end{aligned}
$$

This has the same variance as the BLUE estimator. In the case of unknown hyperparameters, as for the known case, no factorization that enables us to use the RBLS theorem can be found. In this case, we propose the following unbiased estimator

$$
\begin{aligned}
\hat{x}^{\text {Rayleigh }}\left(y_{1: N}\right) & =\frac{\sqrt{N}}{\sqrt{N}-1} y_{(1)}-\frac{1}{N(\sqrt{N}-1)} \sum_{k=1}^{N} y_{k} \\
& =\frac{1}{\sqrt{N}-1}\left(\sqrt{N} y_{(1)}-\bar{y}\right) .
\end{aligned}
$$

Asymptotically, for large $N$, the sample mean and minimum order statistic are independent and the estimator MSE is given by

$$
\operatorname{MSE}\left(\hat{x}^{\text {Rayleigh }}\left(y_{1: N}\right)\right)=\frac{(1+N)(4-\pi) \beta^{2}}{2 N(\sqrt{N}-1)^{2}} .
$$

\section{Mixture of Normal and Uniform Noise Distribution}

Finally, we consider a unique mixture distribution that might be of interest for error modeling in some localization applications. An estimator of the unknown parameter $x$ is derived when the measurement noise is distributed as

$$
e_{k} \sim \alpha \mathcal{N}\left(0, \sigma^{2}\right)+(1-\alpha) \mathcal{U}[0, \beta],
$$

with $\alpha$ denoting the mixing probability of the mixture distribution. Define $f^{\mathcal{U}, \mathcal{N}}\left(y_{k}\right)$ as the probability density function of the considered mixture distribution given by

$$
\begin{aligned}
& f^{\mathcal{U}, \mathcal{N}}\left(y_{k} ; x, \alpha, \sigma^{2}, \beta\right)= \\
& \begin{cases}\frac{\alpha}{\sqrt{2 \pi \sigma^{2}}} \exp \left[-\frac{\left(y_{k}-x\right)^{2}}{2 \sigma^{2}}\right]+\frac{1-\alpha}{\beta} & 0 \leq y_{k}-x \leq \beta \\
\frac{\alpha}{\sqrt{2 \pi \sigma^{2}}} \exp \left[-\frac{\left(y_{k}-x\right)^{2}}{2 \sigma^{2}}\right] & \text { Otherwise. }\end{cases}
\end{aligned}
$$

The BLUE, in the case of the mixture of normal and uniform measurement noise, is given by

$$
\begin{aligned}
\hat{x}_{\mathrm{BLUE}}^{\mathcal{U}, \mathcal{N}}\left(y_{1: N}, \alpha, \beta, \sigma^{2}\right) & =\frac{1}{N} \sum_{k=1}^{N} y_{k}-\frac{\beta(1-\alpha)}{2}, \\
\operatorname{MSE}\left(\hat{x}_{\mathrm{BLUE}}^{\mathcal{U}, \mathcal{N}}\left(y_{1: N}, \alpha, \beta, \sigma^{2}\right)\right) & = \\
& \frac{\beta^{2}(1+(2-3 \alpha) \alpha)+12 \alpha \sigma^{2}}{12 N} .
\end{aligned}
$$

Noting that at $y_{k}-x=0$ the contributions of the uniform distribution and the mean (mode) of the normal distribution are added together, (43) is maximized at this point. The order statistics PDF for $0 \leq y-x \leq \beta$ is given by

$$
\begin{aligned}
f_{(k, N)}^{\mathcal{U}, \mathcal{N}}\left(y ; \alpha, \beta, \sigma^{2}, x, k\right)= \\
N\left(\begin{array}{c}
N-1 \\
k-1
\end{array}\right)\left(\frac{\alpha \exp \left(-\frac{(y-x)^{2}}{2 \sigma^{2}}\right)}{\sqrt{2 \pi \sigma^{2}}}+\frac{1-\alpha}{\beta}\right) \\
\quad \times\left(\frac{(1-\alpha)(y-x)}{\beta}+\frac{\alpha}{2}\left(1+\operatorname{Erf}\left[\frac{y-x}{\sqrt{2 \sigma^{2}}}\right]\right)\right)^{k-1} \\
\quad \times\left(1+\frac{(\alpha-1)(y-x)}{\beta}-\frac{\alpha}{2}\left(1+\operatorname{Erf}\left[\frac{y-x}{\sqrt{2} \sigma}\right]\right)\right)^{N-k}
\end{aligned}
$$

where $\operatorname{Erf}(\cdot)=\frac{2}{\sqrt{\pi}} \int_{0}^{\cdot} e^{-t^{2}} d t$ is the error function. In order to find the best order statistic estimator, we maximize the likelihood function $\ell\left(k \mid y=x, a, \beta, \sigma^{2}\right)$

$$
\begin{aligned}
\ell\left(k \mid y=x, \alpha, \beta, \sigma^{2}\right) & =N\left(\begin{array}{c}
N-1 \\
k-1
\end{array}\right) 2(2-\alpha)^{-k}\left(1-\frac{\alpha}{2}\right)^{N} \alpha^{k-1} \\
& \times\left(\frac{1-\alpha}{\beta}+\frac{\alpha}{\sqrt{2 \pi} \sigma}\right) .
\end{aligned}
$$

Noting that $\left(\frac{1-\alpha}{\beta}+\frac{\alpha}{\sqrt{2 \pi} \sigma}\right)$ is always positive and independent of $k$, we extract it from the likelihood function. Simplifying (46a) by means of manipulating the terms, we get

$$
\begin{aligned}
2(2-\alpha)^{-k} & =2^{1-k}\left(1-\frac{\alpha}{2}\right)^{-k}, \\
\alpha^{k-1} & =\left(2 \frac{\alpha}{2}\right)^{k-1}=2^{k-1}\left(\frac{\alpha}{2}\right)^{k-1} .
\end{aligned}
$$

the likelihood function to be maximized can be re-written as

$$
\ell\left(k \mid y=x, \alpha, \sigma^{2}\right) \propto\left(\begin{array}{c}
N-1 \\
k-1
\end{array}\right)\left(\frac{\alpha}{2}\right)^{k-1}\left(1-\frac{\alpha}{2}\right)^{N-k} .
$$

In order to find the maximum likelihood estimate $\hat{k}=$ arg $\max _{k} \ell(k \mid y-x=0)$, we note that (46d) is a binomial 
TABLE II: Estimators and their MSEs derived for multiple noise distributions.

\begin{tabular}{|c|c|c|}
\hline $\begin{array}{c}\text { Noise } \\
\text { distribution }\end{array}$ & Estimator & MSE \\
\hline $\mathcal{U}[0, \beta]$ & $\begin{array}{l}\hat{x}_{\mathrm{BLUE}}^{\mathcal{U}}\left(y_{1: N}, \beta\right)=\frac{1}{N} \sum_{k=1}^{N} y_{k}-\frac{\beta}{2} \\
\hat{x}_{\mathrm{MVU}}^{\mathcal{U}}\left(y_{1: N}, \beta\right)=\frac{1}{2}\left(y_{(1)}+y_{(N)}\right)-\frac{\beta}{2} \\
\hat{x}_{\mathrm{MVU}}^{\mathcal{U}}\left(y_{1: N}\right)=\frac{N}{N-1} y_{(1)}-\frac{1}{N-1} y_{(N)}\end{array}$ & $\begin{array}{c}\frac{\beta^{2}}{12 N} \\
\frac{\beta^{2}}{2 N(N+3)+4} \\
\frac{N \beta^{2}}{(N+2)\left(N^{2}-1\right)}\end{array}$ \\
\hline $\operatorname{Exp}(\beta)$ & $\begin{array}{l}\hat{x}_{\mathrm{BLUE}}^{\operatorname{Exp}}\left(y_{1: N}, \beta\right)=\frac{1}{N} \sum_{k=1}^{N} y_{k}-\beta \\
\hat{x}_{\mathrm{MVU}}^{\operatorname{Exp}}\left(y_{1: N}, \beta\right)=y_{(1)}-\frac{\beta}{N} \\
\hat{x}_{\mathrm{MVU}}^{\operatorname{Exp}}\left(y_{1: N}\right)=\frac{N}{N-1} y_{(1)}-\frac{\sum_{k=1}^{N} y_{k}}{N(N-1)}\end{array}$ & $\begin{array}{c}\frac{\beta^{2}}{N} \\
\frac{\beta^{2}}{N^{2}} \\
\frac{(N+1) \beta^{2}}{N(N-1)^{2}}\end{array}$ \\
\hline Rayleigh $(\beta)$ & $\begin{array}{l}\hat{x}_{\mathrm{BLUE}}^{\text {Rayleigh }}\left(y_{1: N}, \beta\right)=\frac{1}{N} \sum_{k=1}^{N} y_{k}-\sqrt{\frac{\pi}{2}} \beta \\
\hat{x}^{\text {Rayleigh }}\left(y_{1: N}, \beta\right)=y_{(1)}-\frac{\sqrt{\pi} \beta}{\sqrt{2 N}} \\
\hat{x}^{\text {Rayleigh }}\left(y_{1: N}\right)=\frac{\sqrt{N}}{\sqrt{N}-1} y_{(1)}-\frac{\sum_{k=1}^{N} y_{k}}{N(\sqrt{N}-1)}\end{array}$ & $\begin{array}{c}\frac{(4-\pi) \beta^{2}}{2 N} \\
\frac{(4-\pi) \beta^{2}}{2 N} \\
\frac{(1+N)(4-\pi) \beta^{2}}{2 N(\sqrt{N}-1)^{2}}\end{array}$ \\
\hline$\alpha \mathcal{N}\left(0, \sigma^{2}\right)+(1-\alpha) \mathcal{U}[0, \beta]$ & $\begin{array}{l}\hat{x}_{\mathrm{BLUE}}^{\mathcal{U}, \mathcal{N}}\left(y_{1: N}, \alpha, \beta\right)=\frac{1}{N} \sum_{k=1}^{N} y_{k}-\frac{\beta(1-\alpha)}{2} \\
\hat{x}^{\mathcal{U}, \mathcal{N}}\left(y_{1: N}, \alpha, \beta\right)=y_{\left(\left\lfloor\frac{N \alpha}{2}\right\rfloor+1\right)}\end{array}$ & $\begin{array}{c}\frac{\beta^{2}(1+(2-3 \alpha) \alpha)+12 \alpha \sigma^{2}}{12 N} \\
\text { Unknown }\end{array}$ \\
\hline
\end{tabular}

TABLE III: Bias and MSE of minimum order statistics estimators $\hat{x}_{\text {min }}^{p_{e}}$.

\begin{tabular}{c|c|c}
\hline Distribution & Bias & MSE \\
\hline $\mathcal{U}[0, \beta]$ & $\frac{\beta}{N+1}$ & $\frac{2 \beta^{2}}{(N+1)(N+2)}$ \\
\hline $\operatorname{Exp}(\beta)$ & $\frac{\beta}{N}$ & $\frac{2 \beta^{2}}{N^{2}}$ \\
\hline Rayleigh $(\beta)$ & $\frac{\sqrt{\pi} \beta}{\sqrt{2 N}}$ & $\frac{2 \beta^{2}}{N}$ \\
\hline
\end{tabular}

distribution with probability of success $\frac{\alpha}{2}$. Hence, the maximum of the function is given at the mode of the distribution,

$$
\hat{k}=\left\lfloor\frac{N \alpha}{2}\right\rfloor+1 \quad \text { or }\left\lceil\frac{N \alpha}{2}\right\rceil .
$$

This gives the best order statistic estimator for the case when noise is a mixture of normal and uniform distribution as

$$
\hat{x}^{\mathcal{U}, \mathcal{N}}\left(y_{1: N}, \alpha\right)=y_{(\hat{k})} .
$$

The estimators derived in this section for different noise distributions together with their MSEs are summarized in Tables II and III.

\section{GNSS TIME ESTIMATION}

The precision timing problem using GNSS pseudorange measurements is studied. Given a fixed receiver with known position and $N$ measurements collected from satellites with known positions, the exact time of reception is estimated. The performance of the proposed estimators is evaluated in a simulation study.
Let $p_{t}=\left[p_{t}^{x}, p_{t}^{y}, p_{t}^{z}\right]^{T}$ denote the three-dimensional location of the GNSS receiver and $s_{k, t}=\left[s_{k, t}^{x}, s_{k, t}^{y}, s_{k, t}^{z}\right]^{\top}$ denote the known position of the $k$ :th satellite at time $t$ with $k \in[1, \ldots, N]$. The satellite measurements of pseudorange between the receiver and the $k$ th satellite, $r_{k, t}$ can be modeled as

$$
r_{k, t}=\ell\left(p_{t}, s_{k, t}\right)+x_{t}-\epsilon_{k, t}+e_{k, t},
$$

where $\ell\left(p_{t}, s_{k, t}\right)=\left\|p_{t}-s_{k, t}\right\|_{2}, \mathbb{R}^{3} \rightarrow \mathbb{R}$ gives the Euclidean distance between the receiver and the satellites, $\epsilon_{k, t} \in \mathbb{R}$ and $x_{t} \in \mathbb{R}$ are the satellites' and receiver's clock biases translated into distances, respectively.

Given the satellite position $s_{k, t}$ and satellite clock error $\epsilon_{k, t}$, the unknown bias $x_{t}$ could be estimated using any of the estimators derived in Section III.

$$
\left[\begin{array}{c}
y_{1, t} \\
\vdots \\
y_{N, t}
\end{array}\right]=\left[\begin{array}{c}
r_{1, t}-\ell\left(p_{t}, s_{1, t}\right)+\epsilon_{1, t} \\
\vdots \\
r_{N, t}-\ell\left(p_{t}, s_{N, t}\right)+\epsilon_{N, t}
\end{array}\right] .
$$

In order to have the same geometric dilution of precision (GDOP), in the simulation setup we use the same number of visible satellites with the same locations as in the real data explained in the next section.A total number of 200 epochs $(t=1, \ldots, 200)$ of pseudoranges are generated. For each noise distribution, $M=5000$ Monte Carlo runs are used, and in each Monte Carlo run noise realizations are generated from the four considered distributions with the following hyperparameters

- $e_{t} \sim \mathcal{U}[0,50]$

- $e_{t} \sim \operatorname{Exp}(14)$ 


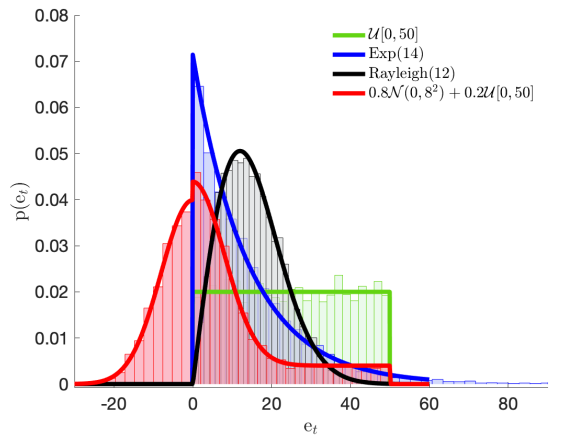

Fig. 1: Measurement error distributions fitted to the generated noise realizations used in the simulations.

- $e_{t} \sim$ Rayleigh $(12)$

- $e_{t} \sim 0.8 \mathcal{N}\left(0,8^{2}\right)+0.2 \mathcal{U}[0,50]$

Fig. 1 illustrates the histogram of the noise realizations of these four measurement errors and the fitted distributions.

Let $\hat{x}_{t}$ denote the estimated receiver clock bias. For each noise distribution, the estimators' performances are evaluated in terms of MSE and root mean squared error (RMSE). The theoretical MSE of each estimator, as given in Table II and Table III, is compared to the numerical MSE obtained in simulations.

Let $\hat{x}_{t}^{(m)}, m=1, \ldots, M$, denote the estimated receiver clock bias at time $t$ in the $m$ th repetition and $\mathbb{E}\left[\hat{x}_{t}\right]=\frac{1}{M} \sum_{m=1}^{M} \hat{x}_{t}^{(m)}$. Define

$$
\begin{aligned}
\hat{b}_{t} & =\mathbb{E}\left[\hat{x}_{t}\right]-x_{t} \\
\hat{\sigma}_{t}^{2} & =\frac{1}{M} \sum_{m=1}^{M}\left(\hat{x}_{t}^{(m)}-\mathbb{E}\left[\hat{x}_{t}\right]\right)^{2} .
\end{aligned}
$$

The numerical MSE at each time $t$ is then computed by

$$
\operatorname{MSE}\left(\hat{x}_{t}\right)=\hat{\sigma}_{t}^{2}+\hat{b}_{t}^{2} \text {. }
$$

For each noise distribution, the theoretical MSEs, marked with solid lines, and numerical MSEs, marked with crosses, are presented in Fig. 2. As the results indicate, the proposed estimator with known hyperparameters of the noise distribution, typically, has less MSE compared to the other estimators. In the case of Rayleigh noise distribution, as expected according to Table II, BLUE and the proposed unbiased estimator give the same variance. Additionally, the theoretical MSEs for the exponential and the Rayleigh noise distributions were obtained asymptotically. Since the number of available satellites at each epoch is $N \in[6,11]$, the theoretical MSE and numerical results do not match. However, as shown in [47], for larger number of sample sizes, the differences are negligible.

The estimation error, in terms of RMSE for the whole duration of each epoch $t=1, \ldots, \tau$ in each Monte Carlo run $m$ is given by

$$
\operatorname{RMSE}\left(\hat{x}_{t}^{(m)}\right)=\sqrt{\frac{1}{\tau} \sum_{t=1}^{\tau}\left(\hat{x}_{t}^{(m)}-x_{t}\right)^{2}} .
$$

The distribution of the $\operatorname{RMSE}\left(\hat{x}_{t}^{(m)}\right)$ is shown in Fig. 3 . The box levels are the $5 \%, 25 \%, 50 \%, 75 \%$, and $95 \%$

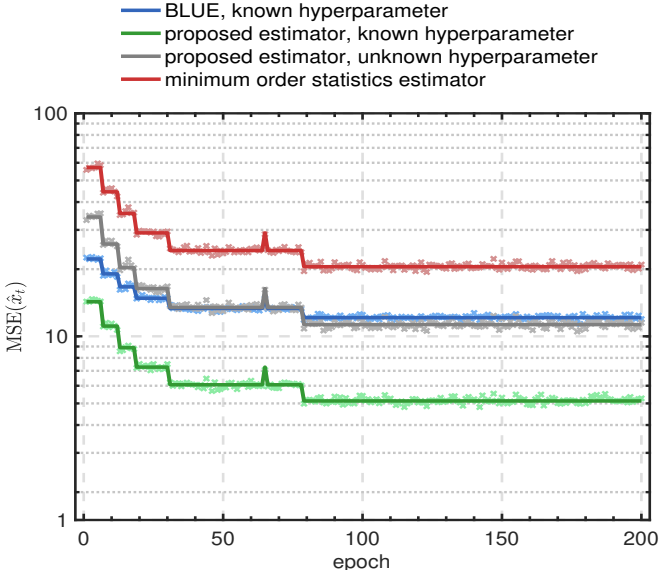

(a) $\mathcal{U}(0,50)$

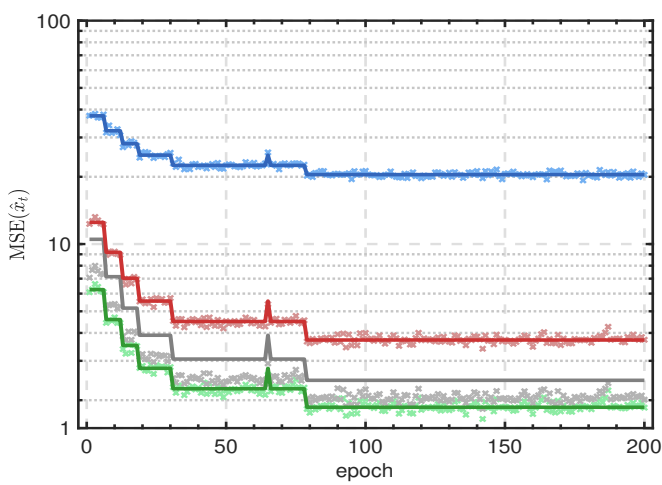

(b) $\operatorname{Exp}(14)$

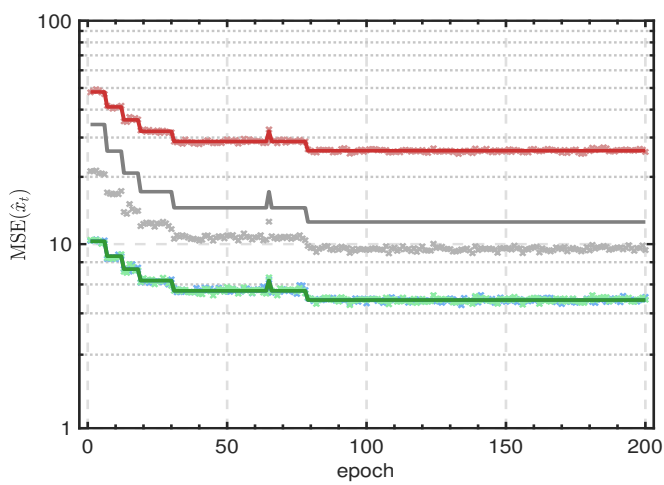

(c) Rayleigh(12)

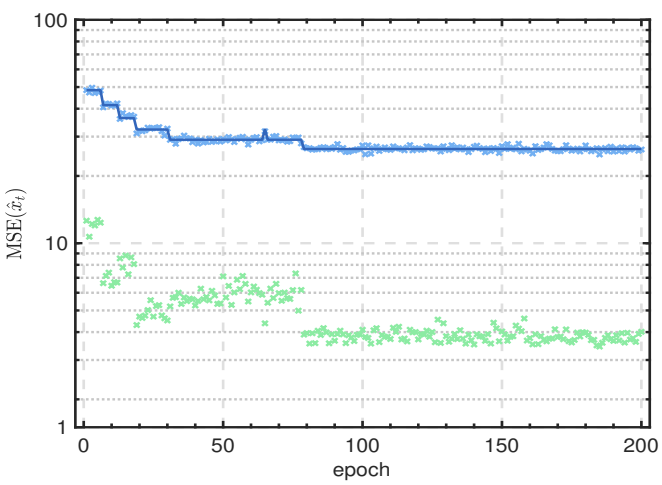

(d) $0.8 \mathcal{N}\left(0,8^{2}\right)+0.2 \mathcal{U}(0,50)$

Fig. 2: Theoretical MSE, marked with solid line, and numerical MSE, marked with crosses, obtained from 5000 Monte Carlo repetitions. 


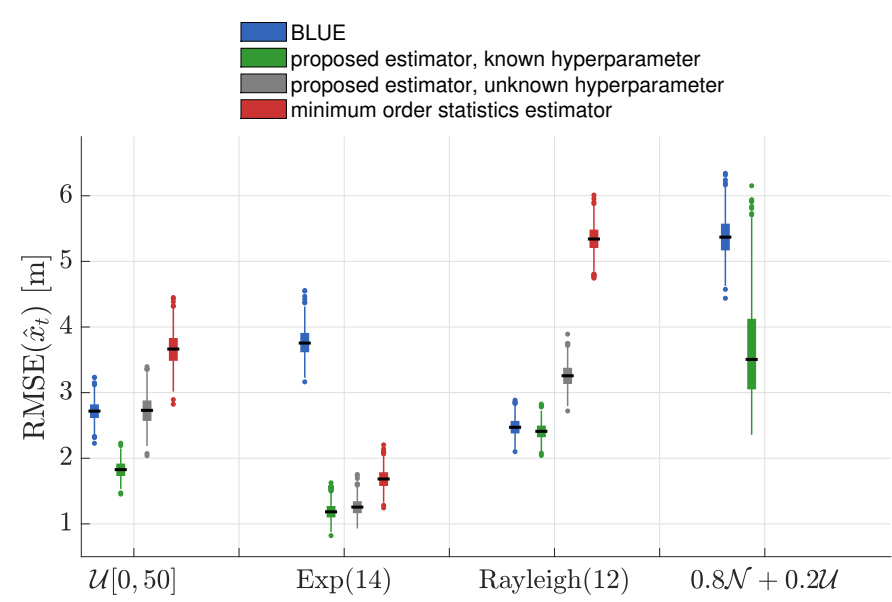

Fig. 3: The distribution of $\operatorname{RMSE}\left(\hat{x}_{t}^{(m)}\right)$.

quantiles and the asterisks show outlier values. As the results indicate, for all noise distributions, the proposed estimator with known hyperparameter of the distribution outperforms the other estimators. In the case of Rayleigh distribution, BLUE and the estimator with known hyperparameter give comparable results. In case of Exponential noise, the MVUE with unknown hyperparameter of the underlying distribution gives roughly the same result as the MVUE with known hyperparameter while BLUE results in large estimation errors.

\section{ITERATIVE GNSS LOCALIZATION}

In the previous section, assuming that the receiver's position $p_{t}$ is known, the clock bias $x_{t}$ was estimated. In this section we extend the problem and propose an iterative approach for joint clock bias and receiver position estimation. Given the estimated position at each time $t$, initialized at $\hat{p}_{0}$, the receiver clock bias $\hat{x}_{t \mid \hat{p}_{t-1}}$ is estimated using the estimators proposed in the previous section. The estimated clock bias is then used to compute $\hat{p}_{t \mid \hat{x}_{t}}$.

Given $k=1, \ldots, N$ pseudorange measurements and satellite clock biases, define $\bar{r}_{k, t}=r_{k, t}+\epsilon_{k, t}$. Similar to (50), using the estimated receiver position, $\hat{p}_{t-1}$, we form $\bar{y}_{k, t}=x_{t}+e_{k, t}$ where

$$
\left[\begin{array}{c}
\bar{y}_{1, t} \\
\vdots \\
\bar{y}_{N, t}
\end{array}\right]=\left[\begin{array}{c}
\bar{r}_{1, t}-\ell\left(\hat{p}_{t-1}, s_{1, t}\right) \\
\vdots \\
\bar{r}_{N, t}-\ell\left(\hat{p}_{t-1}, s_{N, t}\right)
\end{array}\right] .
$$

The estimators derived in Sec. III are employed to find $\hat{x}_{t}$. The estimated receiver clock bias is then used to form the residuals

$$
e_{k, t}=\bar{y}_{k, t}-\hat{x}_{t}, \quad k=1, \ldots, N .
$$

Using a modified Thompson Tau test [53], the measurements giving high residuals are detected and rejected. Let $\tilde{N}$ denote the number of remaining satellites.

Define $S_{t}=\left\{s_{k, t}\right\}_{k \in \tilde{N}} \in \mathbb{R}^{3 \times \tilde{N}}$ denoting the location of the $\tilde{N}$ satellites at time $t$ and $\tilde{\ell}\left(p_{t}, S_{t}\right)=\left\{\ell\left(p_{t}, s_{k, t}\right)\right\}_{k \in \tilde{N}}$, $\mathbb{R}^{3 \times \tilde{N}} \rightarrow \mathbb{R}^{\tilde{N}}$ denoting the vector valued function giving the Euclidean distance between the $\tilde{N}$ satellites and the receiver.

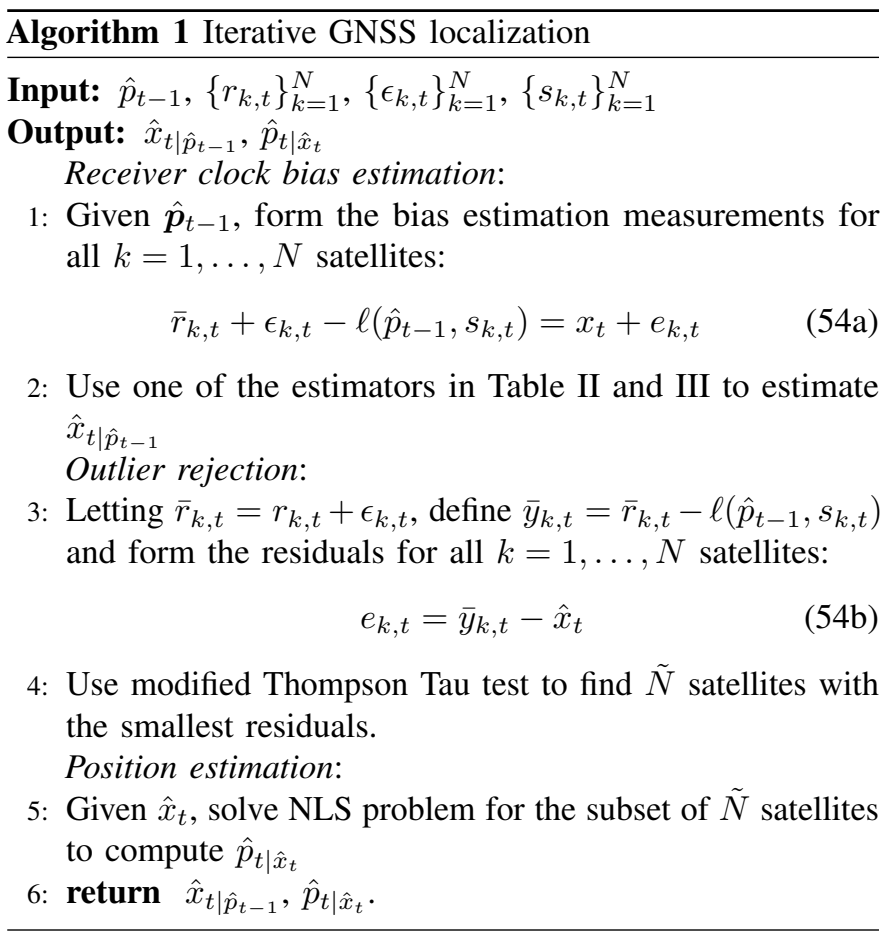

The nonlinear least squares (NLS) cost function for this subset of satellites is given by

$$
\begin{aligned}
V^{\mathrm{NLS}}\left(p_{t}\right)= & \left(\tilde{r}_{1: \tilde{N}, t}-\hat{x}_{1: \tilde{N}, t}-\tilde{\ell}\left(p_{t}, S_{t}\right)\right)^{\top} \\
& \times\left(\tilde{r}_{1: \tilde{N}, t}-\hat{x}_{1: \tilde{N}, t}-\tilde{\ell}\left(p_{t}, S_{t}\right)\right),
\end{aligned}
$$

where $\hat{x}_{1 \tilde{N}, t}=\mathbb{1} \cdot \hat{x}_{t}$ is a vector of size $\tilde{N}$ and $\tilde{r}_{1: \tilde{N}, t}=$ $\left[\bar{r}_{1, t}, \ldots, \bar{r}_{\tilde{N}, t}\right]$. The optimization problem given by the cost function (53c) is solved using the Gauss-Newton algorithm. Algorithm 1 summarizes one iteration of the proposed method.

The local search algorithms, in general, require good initialization, otherwise there is a risk of reaching a local minimum in the loss function $V^{\mathrm{NLS}}\left(p_{t}\right)$. In order to initialize the algorithm and find $\hat{p}_{0}$, we first use the separable least squares (SLS) method described in [54], in which the model (49) is conditionally linear in $x_{t}$, given that $p_{t}$ is known.

Given $N$ visible satellite positions and their pseudoranges and clock errors at time $t=1$, we rewrite the batch formulation of the conditionally linear model $\bar{r}_{1: \tilde{N}, t}=\tilde{\ell}\left(p_{t}, S_{t}\right)+\tilde{\ell}^{l} x_{t}+e_{t}$ where $\tilde{\ell}^{l}=\mathbb{1} \in \mathbb{R}^{N}$. The NLS cost function to be solved to initialize the proposed iterative algorithm is given by

$$
\begin{aligned}
\hat{x}_{1}(p) & =\left(\tilde{\ell}^{l, \top} \tilde{\ell}^{l}\right)^{-1} \tilde{\ell}^{l, \top}\left(\bar{r}_{1: \tilde{N}, 1}-\tilde{\ell}\left(p, S_{1}\right)\right) \\
V_{0}^{\mathrm{NLS}}(p)= & \left(\bar{r}_{1: \tilde{N}, 1}-\tilde{\ell}\left(p, S_{1}\right)-\hat{x}_{1}(p)\right)^{\top} \\
& \times\left(\bar{r}_{1: \tilde{N}, 1}-\tilde{\ell}\left(p, S_{1}\right)-\hat{x}_{1}(p)\right) .
\end{aligned}
$$

An initial value for the receiver's position $\hat{p}_{0}$ is computed by the Gauss-Newton algorithm applied to (55b)

$$
\hat{p}_{0}=\underset{p}{\arg \min } V_{0}^{\mathrm{NLS}}(p) .
$$

The estimated initial position is further used to find $\hat{x}_{1 \mid \hat{p}_{0}}$. We evaluate the performance of the iterative clock bias and 

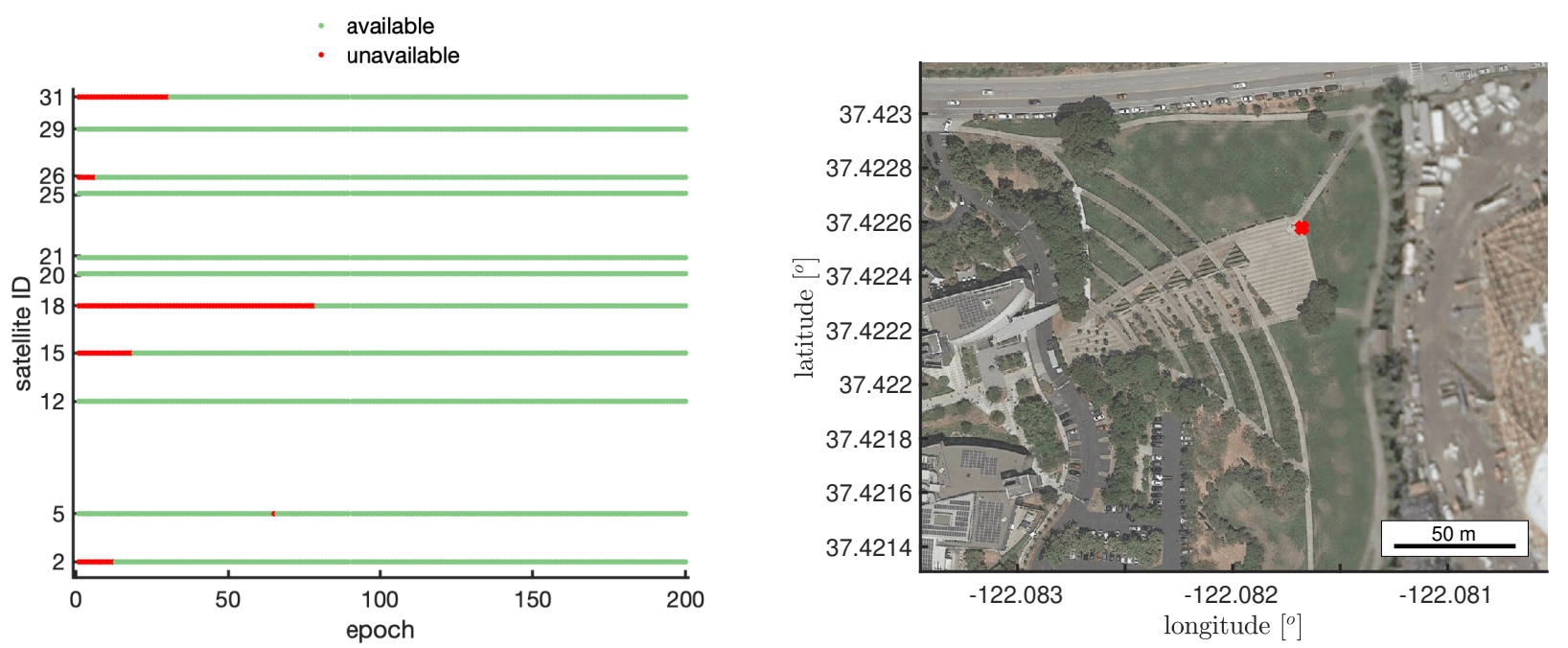

Fig. 4: GNSS measurement with varying number of available satellites during the epoch collected at Charleston Park Test Site. [Top] satellite availability during the epoch [Bottom] the true position of the receiver, marked with red

position estimation procedure using real experimental data in terms of RMSE

$$
\operatorname{RMSE}\left(\hat{p}_{t} \mid \hat{x}_{t}\right)=\sqrt{\frac{1}{\tau} \sum_{t=1}^{\tau}\left\|\hat{p}_{t \mid \hat{x}_{t}}-p_{t}\right\|^{2}} .
$$

The GNSS positioning data set is provided by Google as a demo file in the GPS measurement tools project on GitHub ${ }^{1}$. The data includes pseudorange measurements, satellite positions and satellite clock error. The length of the data is 200 epochs, during which six to eleven satellites are visible to the receiver. Fig. 4a illustrates satellite IDs and their availability, marked with green, and unavailability, marked with red, during the whole epoch. The static receiver's true position is at Charleston Park Test Site, Fig. 4b, whose true latitude, longitude and altitude is available in the dataset.

Since the underlying noise distribution is unknown, we employ all the estimators in Table II which are independent of noise hyperparameters and the minimum order statistic estimator. Let $\hat{x}_{t \mid \hat{p}_{t-1}}^{\mathcal{U}}$ denote the receiver clock bias estimated at time $t$ when the underlying noise is assumed to follow uniform distribution and the estimator with unknown hyperparameter of uniform noise is used. $\hat{p}_{t \mid \hat{x}_{t}}^{\mathcal{U}}$ then means the estimated location of the receiver with the same assumption of the underlying noise. Similarly, define $\hat{p}_{t \mid \hat{x}_{t}}^{\mathrm{exp}}, \hat{p}_{t \mid \hat{x}_{t}}^{\mathrm{ray}}$, and $\hat{p}_{t \mid \hat{x}_{t}}^{\min }$ position estimates for exponential, Rayleigh and minimum order statistic estimators. Additionally, to evaluate the estimator for mixture noise distribution, we let the mixing probability $\alpha=0.9$ and denote its estimate position by $\hat{p}_{t \mid \hat{x}_{t}}^{\text {mix }}$.

In order to evaluate the performance of the proposed estimators and the localization algorithm, we compare the results with two additional methods. In one method, we assume that the underlying noise at each time instance is normally distributed, $e_{t} \sim \mathcal{N}\left(0_{k \times 1}, R_{t}\right)$, where $R_{t} \in \mathbb{R}^{k \times k}$ is the

\footnotetext{
${ }^{1}$ This dataset and the GNSS logging code are maintained on GitHub at the following link: https://github.com/google/gps-measurement-tools
}

TABLE IV: The $95 \%$ percentile RMSEs for real GNSS data.

\begin{tabular}{c|c|c|c|c|c|c|c} 
& $\hat{p}_{t \mid \hat{x}_{t}}^{\mathcal{U}}$ & $\hat{p}_{t \mid \hat{x}_{t}}^{E x p}$ & $\hat{p}_{t \mid \hat{x}_{t}}^{\text {Ray }}$ & $\hat{p}_{t \mid \hat{x}_{t}}^{\min }$ & $\hat{p}_{t \mid \hat{x}_{t}}^{\operatorname{mix}}$ & $\hat{p}_{t \mid \hat{x}_{t}}^{\mathcal{N}}$ & $\hat{\boldsymbol{p}}_{t}^{S L S}$ \\
\hline RMSE $_{\text {Horizontal }}[\mathrm{m}]$ & 10.7 & 9.7 & 12 & 8.9 & 6.9 & 13.5 & 12.5 \\
\hline RMSE $_{\text {Vertical }}[\mathrm{m}]$ & 9.1 & 8.4 & 10.4 & 8.3 & 7.5 & 10.8 & 10.4
\end{tabular}

measurement noise covariance. Google's GNSS logging toolbox is employed to extract satellite measurement noise standard deviations, which is further used to form the diagonal weighting matrix $R$. Following the procedure outlined in Algorithm 1, given $\hat{p}_{t-1}$, we use a standard weighted least square method to estimate $\hat{x}_{t \mid \hat{p}_{t-1}}^{\mathcal{N}}$. The estimated bias is then used to compute $\hat{p}_{t \mid \hat{x}_{t}}^{\mathcal{N}}$. As for other estimators, the algorithm is initialized using the separable least squares method.

Additionally, including the bias inside the state vector and defining $\Psi_{t}=\left[p_{t}, x_{t}\right]$, it is possible to use SLS directly. The SLS problem is solved sequentially in which the estimates at time $t-1$ are used as initial values for time $t$. In order to distinguish between the estimated positions using SLS and those obtained using the proposed iterative algorithm, the dependency on the estimated bias in the subscript is removed. That is, we let $\hat{p}_{t}^{\mathrm{SLS}}$ denote the estimated position at time $t$. The CDF plot of horizontal and vertical positioning errors obtained from different methods is shown in Fig. 5. As the result indicates, using the proposed iterative method and each of the proposed estimators improves both horizontal and vertical positioning accuracy. In order to compare the results more accurately, the $95 \%$ percentile of horizontal and vertical positioning errors for the real GNSS data is provided in Table IV. The best result is obtained by using the proposed iterative method and using the estimator based on mixture noise distribution with $\alpha=0.8$ mixing probability for estimating receiver clock bias. The final positioning RMSE using this approach is around $6.9 \mathrm{~m}$ for horizontal error and around $7.5 \mathrm{~m}$ for vertical error, $95 \%$ of the time. The worst performance is obtained by assuming normally distributed underlying noise. 

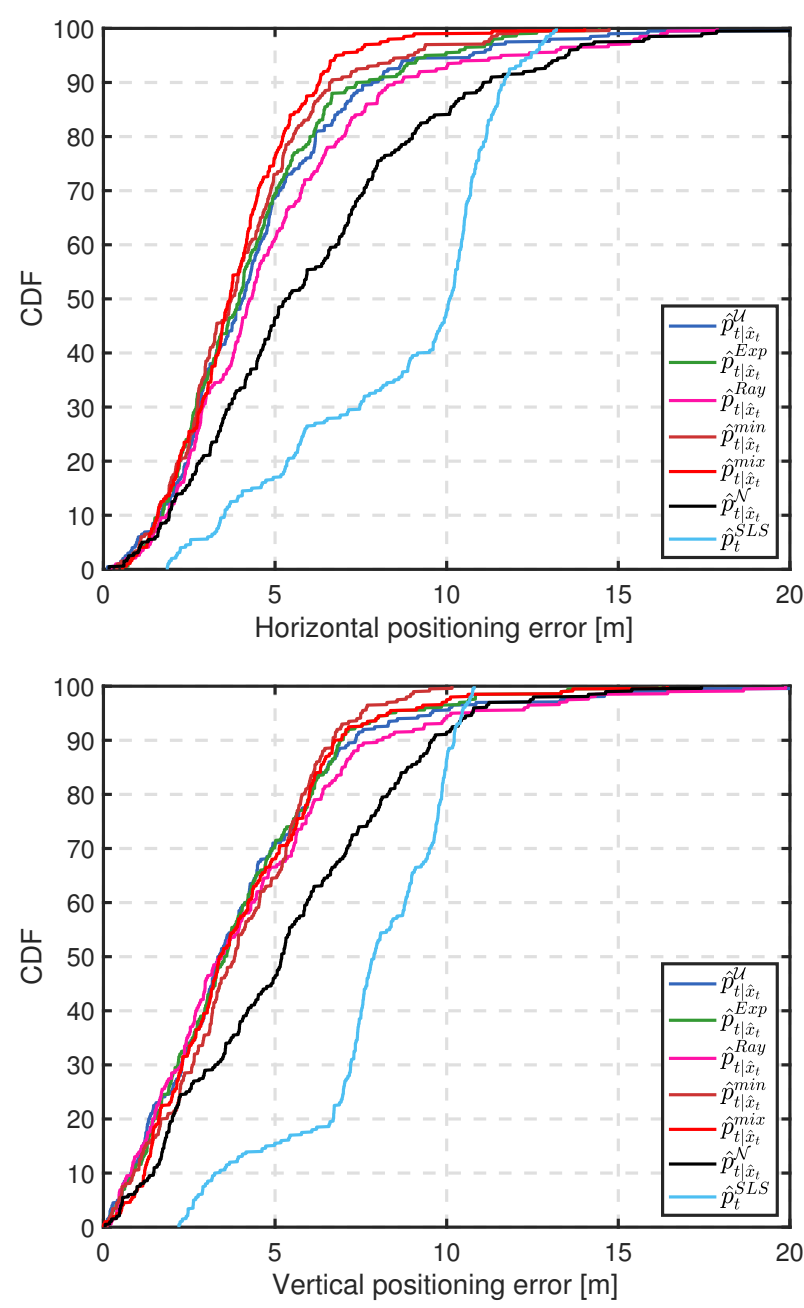

Fig. 5: Empirical error CDFs of horizontal (top) and vertical (bottom) positioning error for real GNSS data using the proposed iterative method and for different estimators of the clock bias as well as the errors obtained using the SLS method.

\section{Vi. Conclusions}

In this work, the location estimation problem was studied in which an unknown parameter was estimated from observations under additive noise. Multiple noise distributions with positive support as well as a mixture of uniform and normal noise distribution were considered. For the considered distributions with positive support, unbiased estimators without any knowledge of the hyperparameters of the underlying noise were also derived. The performance of all the estimators was compared to BLUE and biased minimum order statistic estimator in terms of MSE. Simulations indicate that the proposed estimators outperform BLUE. Additionally, an iterative GNSS localization method was proposed in which the receiver's clock bias was computed using the derived estimators. The experimental data tests with GNSS positioning data indicate the merit of the proposed localization method.

\section{REFERENCES}

[1] S. A. Kassam and H. V. Poor, "Robust techniques for signal processing: A survey," Proceedings of the IEEE, vol. 73, no. 3, pp. 433-481, Mar. 1985.
[2] M. Kok, J. D. Hol, and T. B. Schön, "Indoor positioning using ultrawideband and inertial measurements," IEEE Transactions on Vehicular Technology, vol. 64, no. 4, pp. 1293-1303, Apr. 2015.

[3] B. Chen, C. Yang, F. Liao, and J. Liao, "Mobile location estimator in a rough wireless environment using extended kalman-based IMM and data fusion," IEEE Transactions on Vehicular Technology, vol. 58, no. 3, pp. 1157-1169, Mar. 2009.

[4] F. Gustafsson and F. Gunnarsson, "Mobile positioning using wireless networks: possibilities and fundamental limitations based on available wireless network measurements," IEEE Signal Processing Magazine, vol. 22, no. 4, pp. 41-53, Jul. 2005.

[5] M. Eling, "Fitting insurance claims to skewed distributions: Are the skew-normal and skew-student good models?" Insurance: Mathematics and Economics, vol. 51, no. 2, pp. 239-248, 2012.

[6] R. H. Norden, "A survey of maximum likelihood estimation," International Statistical Review, vol. 40, no. 3, pp. 329-354, 1972.

[7] B. Guermah, H. E. Ghazi, T. Sadiki, Y. Ben Maissa, and E. Ahouzi, "A comparative performance analysis of position estimation algorithms for GNSS localization in urban areas," in Proc. of Advanced Communication Systems and Information Security (ACOSIS), Oct. 2016, pp. 1-7.

[8] D. B. Jourdan, D. Dardari, and M. Z. Win, "Position error bound for UWB localization in dense cluttered environments," IEEE Transactions on Aerospace and Electronic Systems, vol. 44, no. 2, pp. 613-628, 2008.

[9] M. S. Arulampalam, S. Maskell, N. Gordon, and T. Clapp, "A tutorial on particle filters for online nonlinear/non-Gaussian Bayesian tracking," IEEE Transactions on Signal Processing, vol. 50, no. 2, pp. 174-188, 2002.

[10] J. Prieto, S. Mazuelas, A. Bahillo, P. Fernandez, R. M. Lorenzo, and E. J. Abril, "Adaptive data fusion for wireless localization in harsh environments," IEEE Transactions on Signal Processing, vol. 60, no. 4, pp. 1585-1596, 2012.

[11] F. Daum, "Nonlinear filters: beyond the kalman filter," IEEE Aerospace and Electronic Systems Magazine, vol. 20, no. 8, pp. 57-69, 2005.

[12] S. Mazuelas, Y. Shen, and M. Z. Win, "Belief condensation filtering," IEEE Transactions on Signal Processing, vol. 61, no. 18, pp. 4403-4415, 2013.

[13] F. Yin, C. Fritsche, F. Gustafsson, and A. M. Zoubir, "TOA-based robust wireless geolocation and cramér-rao lower bound analysis in harsh LOS/NLOS environments," IEEE Transactions on Signal Processing, vol. 61, no. 9, pp. 2243-2255, May 2013.

[14] Y. T. Chan, W. Y. Tsui, H. C. S., and P. C. Ching, "Time-of-arrival based localization under NLOS conditions," IEEE Transactions on Vehicular Technology, vol. 55, no. 1, pp. 17-24, 2006.

[15] Y. Qi, H. Kobayashi, and H. Suda, "Analysis of wireless geolocation in a non-line-of-sight environment," IEEE Transactions on Wireless Communications, vol. 5, no. 3, pp. 672-681, 2006.

[16] J. Riba and A. Urruela, "A non-line-of-sight mitigation technique based on ml-detection," in Proc. of IEEE International Conference on Acoustics, Speech, and Signal Processing (ICASSP), vol. 2, Montreal, QC, Canada, May 2004, pp. 153-156.

[17] X. Wang, Z. Wang, and B. O'Dea, "A TOA-based location algorithm reducing the errors due to non-line-of-sight (NLOS) propagation," IEEE Transactions on Vehicular Technology, vol. 52, no. 1, pp. 112-116, 2003.

[18] S. Venkatesh and R. M. Buehrer, "A linear programming approach to NLOS error mitigation in sensor networks," in Prof. of International Conference on Information Processing in Sensor Networks (IPSN), Nashville, TN, USA, Apr. 2006, pp. 301-308.

[19] H. Chen, G. Wang, Z. Wang, H. C. So, and H. V. Poor, "Non-line-of-sight node localization based on semi-definite programming in wireless sensor networks," IEEE Transactions on Wireless Communications, vol. 11, no. 1, pp. 108-116, 2012.

[20] R. Casas, A. Marco, J. J. Guerrero, and J. Falco, "Robust estimator for non-line-of-sight error mitigation in indoor localization," EURASIP Journal on Advances in Signal Processing, vol. 2006, no. 1, pp. 1-8, 2006.

[21] Z. Li, W. Trappe, Y. Zhang, and B. Nath, "Robust statistical methods for securing wireless localization in sensor networks," in Proc. of International Symposium on Information Processing in Sensor Networks (IPSN), Los Angeles, CA, USA, Apr. 2005, pp. 91-98.

[22] S. M. Stigler, "Simon Newcomb, Percy Daniell, and the history of robust estimation 1885-1920," Journal of the American Statistical Association, vol. 68 , no. 344, pp. 872-879, 1973.

[23] S. A. Kassam, Signal Detection in Non-Gaussian Noise. Springer-Verlag New York, 1988.

[24] C. Stewart, "Robust parameter estimation in computer vision," SIAM Review, vol. 41, no. 3, pp. 513-537, 1999. 
[25] G. R. Arce, Nonlinear Signal Processing: A Statistical Approach. Hoboken, NJ: Wiley, 2004.

[26] A. M. Zoubir, V. Koivunen, Y. Chakhchoukh, and M. Muma, "Robust estimation in signal processing: A tutorial-style treatment of fundamental concepts," IEEE Signal Processing Magazine, vol. 29, no. 4, pp. 61-80, Jul. 2012.

[27] E. Eskin, "Anomaly detection over noisy data using learned probability distributions," in In Proc. of the International Conference on Machine Learning, Stanford, CA, USA, Jun. 2000, pp. 255-262.

[28] S. Chawla, D. Hand, and V. Dhar, "Outlier detection special issue," Data Mining and Knowledge Discovery, vol. 20, no. 2, pp. 189-190, Mar. 2010.

[29] V. J. Hodge and J. Austin, "A survey of outlier detection methodologies," Artificial Intelligence Review, vol. 22, no. 2, pp. 85-126, Oct. 2004.

[30] C. Fritsche, U. Hammes, A. Klein, and A. M. Zoubir, "Robust mobile terminal tracking in NLOS environments using interacting multiple model algorithm," in Proc. of International Conference on Acoustics, Speech and Signal Processing (ICASSP), Taipei, Taiwan, Apr. 2009, pp. 3049-3052.

[31] H. A. David and H. N. Nagaraja, Encyclopedia of Statistical Sciences. American Cancer Society, 2006, ch. Order Statistics.

[32] Z. Wang and X. Yang, "Ultra wide-band communications with blind channel estimation based on first-order statistics," in Proc. of Acoustics Speech, and Signal Processing, May 2004, pp. iv-iv.

[33] Z. Zheng, J. Sun, W. Q. Wang, and H. Yang, "Classification and localization of mixed near-field and far-field sources using mixed-order statistics," Signal Processing, vol. 143, 2018.

[34] K. Wang, L. Wang, J. Shang, and X. Qu, "Mixed near-field and far-field source localization based on uniform linear array partition," IEEE Sensors Journal, vol. 16, no. 22, pp. 8083-8090, Nov. 2016

[35] H. He, Y. Wang, and J. Saillard, "A high resolution method of source localization in near-field by using focusing technique," in Proc. of European Signal Processing Conference, Aug. 2008, pp. 1-5.

[36] M. Aktas and T. E. Tuncer, "Iterative HOS-SOS (IHOSS) algorithm for direction-of-arrival estimation and sensor localization," IEEE Transactions on Signal Processing, vol. 58, no. 12, pp. 6181-6194, Dec. 2010.

[37] J. Liang and D. Liu, "Passive localization of mixed near-field and farfield sources using two-stage MUSIC algorithm," IEEE Transactions on Signal Processing, vol. 58, no. 1, pp. 108-120, Jan. 2010

[38] J. He, M. N. S. Swamy, and M. O. Ahmad, "Efficient application of MUSIC algorithm under the coexistence of far-field and near-field sources," IEEE Transactions on Signal Processing, vol. 60, no. 4, pp. 2066-2070, Apr. 2012.

[39] K. C. Ho, "Bias reduction for an explicit solution of source localization using tdoa," IEEE Transactions on Signal Processing, vol. 60, no. 5, pp. 2101-2114, May 2012.

[40] S. M. Kay, Fundamentals of Statistical Signal Processing: Estimation Theory. Upper Saddle River, NJ, USA: Prentice-Hall, Inc., 1993.

[41] E. L. Lehmann and G. Casella, Theory of Point Estimation. SpringerVerlag New York, 1998.

[42] H. L. V. Trees, Detection, Estimation, and Modulation Theory: Detection, Estimation, and Linear Modulation Theory. John Wiley \& Sons, 2001.

[43] E. L. Lehmann and H. Scheffé, "Completeness, similar regions, and unbiased estimation: Part I," The Indian Journal of Statistics, vol. 10 no. 4 , pp. $305-340,1950$.

[44] R. A. Fisher and M. A. Phil, "On the mathematical foundations of theoretical statistics," Philosophical Transactions of the Royal Society of London A: Mathematical, Physical and Engineering Sciences, vol. 222, no. 594-604, pp. 309-368, Jan. 1922

[45] P. R. Halmos and L. J. Savage, "Application of the Radon-Nikodym theorem to the theory of sufficient statistics," The Annals of Mathematical Statistics, vol. 20, no. 2, pp. 225-241, Jun. 1949.

[46] U. Kamps, "A concept of generalized order statistics," Journal of Statistical Planning and Inference, vol. 48, no. 1, p. 1, 1995.

[47] K. Radnosrati, G. Hendeby, and F. Gustafsson, "Exploring positive noise in estimation theory," in eprint arXiv: 1910.01569, Dec. 2019.

[48] J. B. McDonald and Y. J. Xu, "A generalization of the beta distribution with applications," Journal of Econometrics, vol. 66, no. 1, pp. 133-152, Mar. 1995.

[49] H. A. David and H. N. Nagaraja, Order Statistics. John Wiley \& Sons, 2004.

[50] S. B. Moon, P. Skelly, and D. Towsley, "Estimation and removal of clock skew from network delay measurements," in Proc. of IEEE Computer and Communications Societies (ICCS), New York, NY, USA, Mar. 1999. pp. 227-234.

[51] T. Trump, "Maximum likelihood trend estimation in exponential noise," IEEE Transactions on Signal Processing, vol. 49, no. 9, pp. 2087-2095, 2001.
[52] R. F. Tate, "Unbiased estimation: Functions of location and scale parameters," Annals of Mathematical Statistics, vol. 30, no. 2, pp. 341366, 1959.

[53] A. J. Wheeler and A. R. Ganji, Introduction to Engineering Experimentation. Pearson Education (US), 2009.

[54] F. Gustafsson, Statistical Sensor Fusion. Professional Publishing House, 2012. 